\title{
Mean distances on a sphere
}

\author{
Clark Kimberling
}

Clark Kimberling received his Ph.D. in mathematics from the Illinois Institute of Technology in 1970. Since 1970 he has been a member of the mathematics department at the University of Evansville, Indiana. His interests include number theory and maintaining the Encyclopedia of Triangle Centers:

http: //faculty.evansville.edu/ck6/encyclopedia/ETC.html.

\section{Introduction}

Consider a set of $m$ points scattered on a sphere. Each such point $P$ has a surrounding "neighborhood" - the open set of points closer to $P$ than to any of the other $m-1$ points. The mean distance from $P$ to its neighborhood is defined in the usual way by integration, and the mean distance from the whole sphere to the nearest of the $m$ points is then the weighted mean of the $m$ neighborhood means.

In this article we estimate such mean distances for various choices of $m$ points, including vertex sets of the five regular polyhedra. Surprisingly, these appear to be new. For example, given a regular tetrahedron inscribed in the unit sphere, the mean distance to the nearest vertex is approximately 0.690737280538164 . In the final section we consider distance as a probability random variable, of which "expected value" is a synonym for mean distance.

We use the standard labeling of a right triangle $A B C$ on the unit sphere with center $O$, with vertex angles labeled $\alpha, \beta, \gamma$ and sidelengths (alias angles $B O C, C O A, A O B$ ) labeled

In der vorliegenden Arbeit geht es um die Bestimmung von mittleren Abständen auf der Kugeloberfläche. Für eine um den Nordpol zentrierte Kugelkappe der nördlichen Hemisphäre wird der mittlere Abstand zum Nordpol durch „Aufsummieren“ (Integration) der infinitesimalen Flächenanteile der Kugelkappe multipliziert mit der Distanz zum Nordpol im Verhältnis zur Gesamtfläche der Kugelkappe berechnet. Wenn man beispielsweise für die Kugelkappe die ganze nördliche Hemisphäre wählt, so ergibt als mittlere Distanz zum Nordpol 1. In Verallgemeinerung zu diesem Ergebnis bestimmt der Autor auch mittlere Abstände für regelmäßige sphärische Polygone zu endlichen Punktmengen der Sphäre. 
$a, b, c$. By Girard's theorem, the surface area of $A B C$ is $\alpha+\beta+\gamma-\pi$, and by Napier's pentagon,

$$
\alpha=\arccos (\tan b \cot c) .
$$

These fundamentals are developed in spherical trigonometry textbooks (e.g., [2], [4]), and graphics, some interactive, are found at many websites (e.g., [1], [3]).

\section{Integrals}

Let $\mathcal{R}$ be a cap or zone on the upper unit hemisphere

$$
z=\sqrt{1-x^{2}-y^{2}},
$$

and let $\mathcal{A}_{\mathcal{R}}$ be the area of $\mathcal{R}$. The mean of a continuous function $f$ on $\mathcal{R}$ is defined by the surface integral

$$
\int_{\mathcal{R}} \frac{1}{\mathcal{A}_{\mathcal{R}}} f(x, y, z) d S .
$$

Suppose $N=(0,0,1)$, the north pole, and consider concentric circles on $\mathcal{R}$, centered at $N$. If $s$ denotes the distance on $\mathcal{R}$ from a point on such a circle to $N$, then the radius of the circle is $\sin s$. Thus, an increment $d s$ accounts for an area $d S=(2 \pi \sin s) d s$, so that the mean distance from $N$ to $\mathcal{R}$ can be found using

$$
\frac{1}{\mathcal{A}_{\mathcal{R}}} \int s d S=\frac{2 \pi}{\mathcal{A}_{\mathcal{R}}} \int s \sin s d s .
$$

The limits of integration are constants, since $\mathcal{R}$ is a cap or zone. For other regions we can find mean distances starting with integrals of the form (3). Certain standard properties of integrals will be needed. Let $M(H, R)$ be the mean distance from a point $H$ to a region $R$, a cap, zone, or spherical triangle.

(I1) If $R^{\prime}$ is a rotation of $R$ about $H$, then $M\left(H, R^{\prime}\right)=M(H, R)$.

(I2) If $R^{\prime}$ is a reflection of $R$ about a line $L$ through $H$, then $M\left(H, R^{\prime}\right)=M(H, R)$.

(I3) If $R^{\prime}$ is a pairwise disjoint union of such rotations and reflections, then $M\left(H, R^{\prime}\right)=$ $M(H, R)$.

(I4) Weighted means: suppose that $n \geq 1$, and that $R^{\prime}$ is the union of pairwise disjoint regions $R_{i}$ having respective areas $a_{i}$ and mean distances $m_{i}=M\left(H, R_{i}\right)$ for $i=$ $1,2, \ldots, n$. Let

$$
s_{1}=a_{1} m_{1}+a_{2} m_{2}+\ldots+a_{n} m_{n} \quad \text { and } \quad s_{2}=a_{1}+a_{2}+\ldots+a_{n} .
$$

Then $M\left(H, R^{\prime}\right)=s_{1} / s_{2}$.

(I5) If $R^{\prime}$ is the interior of $R$, then $M\left(H, R^{\prime}\right)=M(H, R)$.

For example, the mean distance from the center $G$ of a spherical equilateral triangle $A B C$ equals the mean distance from $G$ to the spherical triangle $G B C$. Likewise, we can compute mean distances involving other regular polygons, as in Section 6, and mean distances of right triangles, as in Section 4. First, however, we shall examine somewhat simpler mean distances. 


\section{Caps and zones}

Suppose that $0 \leq a<b \leq 1$. Then on the unit hemisphere (2), the points satisfying $a \leq z \leq b$ comprise a cap if $b=1$ and a zone otherwise. In both cases the area of the region is $2 \pi(b-a)$, and the inequality $a<b$ is equivalent to $\arccos b<s<\arccos a$. With $N=(0,0,1)$, mean distances are found from (3):

$$
M=\frac{1}{b-a} \int_{\arccos b}^{\arccos a} s \sin s d s
$$

so that

$$
M=\frac{\sqrt{1-a^{2}}-\sqrt{1-b^{2}}+b \arccos b-a \arccos a}{b-a} .
$$

If $b=1$ and we write $a$ as a variable $x$, then for caps,

$$
M(x)=\frac{\sqrt{1-x^{2}}-x \arccos x}{1-x} .
$$

Especially notable is the fact that $M(0)=1$; that is, the mean distance from the north pole to the northern hemisphere is simply 1 radian. The locus of a point having actual distance 1 from $N$ is the circle in which the plane $z=\cos 1$ meets the unit sphere. This circle has radius $\sin 1$, and the area of its spherical cap is $2 \pi(1-\cos 1)$, leaving $2 \pi \cos 1$ as the area of the complementary zone down to the plane $z=0$.

Regarding Earth as a unit sphere, the latitude of the circle at distance 1 from the north pole - the mean latitude of the northern hemisphere - is about 32.7 degrees. It passes through or close to Ft. Worth (Texas), San Diego (California), and Nazareth (Israel); see Fig. 1.

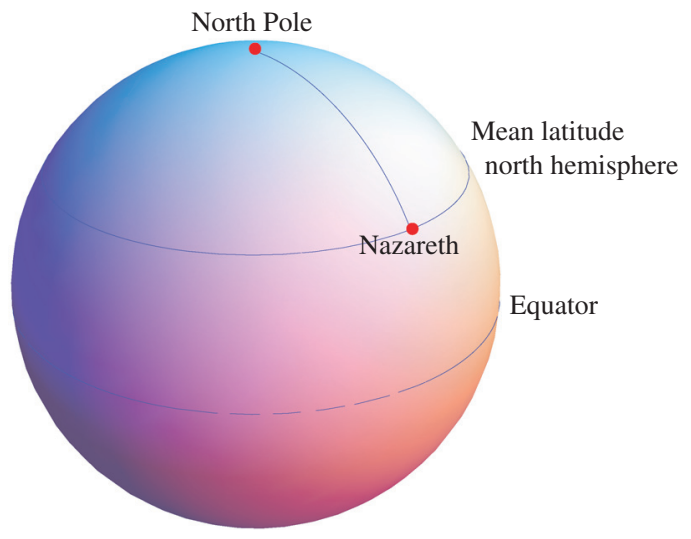

Fig. 1 The distance from the North Pole to Nazareth is 1 radian

If $H$ is a fixed point on the equator and $Z$ is a zone or cap having north pole $N=(0,0,1)$, then the mean distance from $H$ to $Z$ is $\pi / 2$. To see this, rotate the configuration so that $H$ 


\begin{tabular}{|c|c|c|}
\hline$a$ & $b$ & $M$ \\
\hline \hline 0 & 1 & 1.000000000 \\
\hline $1 / 4$ & 1 & 0.851622425 \\
\hline $1 / 2$ & 1 & 0.6848532564 \\
\hline $3 / 4$ & 1 & 0.4775485676 \\
\hline $7 / 8$ & 1 & 0.3354597742 \\
\hline $15 / 16$ & 1 & 0.2364463375 \\
\hline 0 & $1 / 4$ & 1.4451327254 \\
\hline 0 & $1 / 2$ & 1.3151467436 \\
\hline 0 & $3 / 4$ & 1.5082145624 \\
\hline $1 / 3$ & $2 / 3$ & 1.0435370682 \\
\hline $1 / 2$ & 0.5801952659 & 1.0000000000 \\
\hline
\end{tabular}

Table 1 Mean distances from $N$

goes to the north pole and $N$ goes to the equator. Then the image of $Z$ is symmetric about the equator, so that its mean distance to the north pole is $\pi / 2$. For comparison, the mean distance from the whole equator to the whole northern hemisphere is $\pi / 2-1$.

As a supplement to Table 1 , for fixed $H$ and $b=1$, we select values of $M$ and solve for $a$ :

\begin{tabular}{|c|c|c|}
\hline$a$ & $b$ & $M$ \\
\hline \hline 0.930181394058 & 1 & 0.2500000000007 \\
\hline 0.726629934235 & 1 & 0.5000000000006 \\
\hline 0.406913243452 & 1 & 0.7500000000001 \\
\hline 0 & 1 & 1 \\
\hline
\end{tabular}

Table 1A Mean distances from $N$ to caps

If $a=0$ and we treat $b$ as a variable $x$, then the mean distance $\mathcal{M}$ from the north pole $H=(0,0,1)$ to the zone from the equator $a=0$ up to the plane $z=b$ is given via (4) by

$$
\mathcal{M}(x)=\frac{1-\sqrt{1-x^{2}}+x \arccos x}{x} .
$$

As a second supplement to Table 1 , we select values of $\mathcal{M}$ and solve for $b$ : 


\begin{tabular}{|c|c|c|}
\hline$a$ & $b$ & $\mathcal{M}$ \\
\hline \hline 0 & 0.0000000001 & $1.5707968367 \quad(\approx \pi / 2)$ \\
\hline 0 & 0.6190540690 & 1.1250000000 \\
\hline 0 & 0.1413558550 & 1.5000000000 \\
\hline 0 & 1 & 1 \\
\hline
\end{tabular}

Table 1B Mean distances from $N$ to zones

\section{Spherical triangles}

Suppose that $A B C$ is a right spherical triangle labeled with $A, B, C, a, b, c, \alpha, \beta, \gamma$ in the standard way, with vertex angle $\gamma=\pi / 2$ at $C$. Assume that $A B C$ lies in the northern hemisphere (2), that $N=(0,0,1)$ and $O=(0,0,0)$, and let $\mathcal{A}$ denote the area of $A B C$. Noting that $b<c$, let $X$ be the point on the great circle $A C N$ situated so that the arc $A C X$ has length $c$. Then the isosceles triangle $A B X$ is the union of triangles $A B C$ and $C X B$. Consequently, the mean distance $M$ from vertex $A$ to triangle $A B C$ is given by

$$
M=\frac{1}{\mathcal{A}}\left(\int_{0}^{c} \alpha s \sin s d s-\int_{b}^{c} k(s) s \sin s d s\right),
$$

where the arclength $k(s)$ is found as an angle from Napier's pentagon as in (1):

$$
k(s)=\arccos (\tan b \cot s) .
$$

Now suppose that $U V W$ is an equilateral spherical triangle with vertex angle $\alpha$ and sidelength $x \leq \pi / 2$. By Girard's formula, the area, $\mathcal{A}(x)$, of $U V W$ is $3 \alpha-\pi$, and solving for $\alpha$ gives

$$
\mathcal{A}(x)=3 \arccos \left(\frac{\cos x}{1+\cos x}\right)-\pi .
$$

Let $C$ be the midpoint of arc $V W$. Let $A=U$ and $B=V$. Then $A B C$ is a right spherical triangle, and the mean distance from $A$ to $A B C$ equals the mean distance from $U$ to $U V W$. In order to apply (5) to $A B C$, we have by Napier's pentagon, or more explicitly [4, equations (1)-(10), pp. 194-195],

$$
\begin{aligned}
c & =x, \\
\alpha & =\arccos \left(\frac{\cos x}{1+\cos x}\right)=\arcsin (\sin (x / 2) \csc x), \\
b & =\arccos (\sec (x / 2) \cos x), \\
k(s) & =\arccos (\tan b \cot s), \\
\mathcal{A}_{A B C} & =\frac{1}{2} \mathcal{A}(x) .
\end{aligned}
$$

Now (5) gives the following results for $U V W$ : 


\begin{tabular}{|c|c|c|}
\hline sidelength $x$ & area of $U V W$ & $M(U$ to $U V W)$ \\
\hline \hline$\pi / 2 \approx 1.57079$ & $\pi / 2$ & 1.0000000 \\
\hline $\arctan 2 \approx 1.10$ & $\pi / 5 \approx 0.6283$ & 0.6854769 \\
\hline$\pi / 3 \approx 1.04719$ & 0.5512855 & 0.6469076 \\
\hline$\pi / 4 \approx 0.78539$ & 0.2895605 & 0.4815149 \\
\hline$\pi / 6 \approx 0.52359$ & 0.12293598 & 0.3194700 \\
\hline 1.33540100935 & 1 & 0.8358397 \\
\hline 1.00386508622 & $1 / 2$ & 0.6192159 \\
\hline 1.35907989763 & $\pi / 3$ & 0.8518439 \\
\hline 1.21408103950 & $\pi / 4$ & 0.7551357 \\
\hline 1.02417556740 & $\pi / 6$ & 0.6321770 \\
\hline 1 & 0.49559489 & 0.6167529 \\
\hline $1 / 2$ & 0.11175309 & 0.3049730 \\
\hline 0.814978563 & 0.31382189 & 0.5000007 \\
\hline 0.4103134499 & 0.07447024 & 0.2500004 \\
\hline
\end{tabular}

Table 2 Mean distance $M$ from $U$ to equilateral triangle $U V W$

\section{Lunes}

Let $\psi \leq \pi$ be the angle between planes $\mathcal{P}_{1}$ and $\mathcal{P}_{2}$ that pass through the center of the unit sphere $x^{2}+y^{2}+z^{2}=1$. The wedge-shaped region between $\mathcal{P}_{1}$ and $\mathcal{P}_{2}$ is called a lune of angle $\psi$. A lune may be regarded as a 2 -sided spherical polygon. In order to compute mean distances from a point to a lune, we begin by assuming, without loss of generality, that the endpoints of the lune are $(0,-1,0)$ and $(0,1,0)$ and that the lune is bisected by the $x y$-plane. The center of the lune is the point $H=(1,0,0)$. For $0 \leq s<\psi / 2$, a circle about $H$ at distance $s$ has length $2 \pi \sin s$. For $0 \leq s<\psi / 2$, the distance from the plane $z=0$ is $\leq \tan (\psi / 2) \cos s$, so that the remaining arclength is given by

$$
\ell(s)=\arcsin \left(\frac{\tan (\psi / 2)}{\tan s}\right) \sin s .
$$

As this length occurs 4 times, we assemble the mean distance from $H$ to the lune as

$$
M=\frac{1}{2 \psi}\left(2 \pi \int_{0}^{\psi / 2} s \sin s d s+4 \int_{\psi / 2}^{\pi / 2} s \ell(s) d s\right)
$$




\begin{tabular}{|c|c|}
\hline$\psi / 2$ & $M$ \\
\hline \hline$\pi / 2 \approx 1.570796$ & 1.00000000 \\
\hline$\pi / 3 \approx 1.047198$ & 0.81680351 \\
\hline$\pi / 4 \approx 0.785398$ & 0.73335388 \\
\hline$\pi / 6 \approx 0.523599$ & 0.65925289 \\
\hline
\end{tabular}

Table 3 Mean distance $M$ from center $H$ to lune of angle $\psi$

\section{Regular spherical polygons}

Suppose that $P_{k}=V_{1} V_{2} \ldots V_{k}$, for $k \geq 3$, is a regular spherical polygon on a sphere. Let $A$ be the center (on the sphere) of $P_{k}$. Then $V_{i} A V_{i+1}$, for $i=1,2, \ldots, k-1$, as well as $V_{k} A V_{1}$, are congruent isosceles triangles.

Let $C$ be the midpoint of arc $V_{1} V_{2}$, and let $B=V_{1}$. Then $A B C$ is one of $2 k$ congruent right spherical triangles sharing $A$ as a vertex. The angle $V_{1} A V_{2}$ is one of $k$ congruent angles at $A$, so that $\measuredangle V_{1} A V_{2}=2 \pi / k$; consequently $\measuredangle B A C=\pi / k$, a fact to be used in Section 8. By (I3) in Section 2, the mean distance from $A$ to $A B C$ equals the desired mean distance from $A$ to the whole polygon $P_{k}$.

\section{Inscribed polyhedra}

Suppose that $V_{1}, V_{2}, \ldots, V_{k}$ are the vertices of a polyhedron $\mathbb{P}$ inscribed in a sphere $\mathbb{S}$. Define the spherical mean distance, $M(\mathbb{P})$, from $\mathbb{S}$ to $\mathbb{P}$ (or from $\mathbb{P}$ to $\mathbb{S}$ ) as the mean distance from $\mathbb{S}$ to the nearest vertex of $\mathbb{P}$. In order to evaluate $M(\mathbb{P})$, it is helpful to recognize, for each $i$, the neighborhood of $V_{i}$, as defined in Section 1:

$$
N_{i}=\left\{X \in \mathbb{S}: s\left(X, V_{i}\right)<s\left(X, V_{j}\right) \text { for all } j \neq i\right\},
$$

where $s(X, V)$ denotes the distance between the points $X$ and $V$.

To construct $N_{i}$, for each $j \neq i$, let $B_{i j}$ be the perpendicular bisector (a great circle) of the spherical segment $V_{i} V_{j}$. Of the two open hemispheres having boundary $B_{i j}$, let $H_{i j}$ be the one containing $V_{i}$. Then

$$
N_{i}=\bigcap_{j \neq i} H_{i j}
$$

Clearly $N_{i}$ is the interior of a spherical polygon of at most $k$ sides. If $\mathbb{P}$ is regular, as in Section 8, then the polygons $N_{i}$ are regular and congruent, which makes the calculation of $M(\mathbb{P})$ relatively easy. However, before turning to such calculations - in the next section we note that in the general case, it is helpful to regard the construction of a neighborhood one step at a time, as illustrated here for the neighborhood of $V_{1}$ : 
Step 1. Construct the perpendicular bisector $B_{12}$ of segment $V_{1} V_{2}$, so that $H_{12}$ is the open hemisphere containing $V_{1}$. The construction is finished if $k=2$, and in this case $N_{1}=H_{12}$.

Step 2. Otherwise, construct the perpendicular bisector $B_{13}$ of segment $V_{1} V_{2}$, thus obtaining the open hemisphere $H_{13}$ containing $V_{1}$. The construction is finished if $k=3$, and in this case $N_{1}=H_{12} \cap H_{13}$, the interior of a lune. In any case, note that the location of $H_{23}$ plays no part in the determination of $N_{1}$.

Continuation. If $n \geq 4$, then for $4 \leq k \leq n$, the interior of the spherical polygon $H_{12} \cap H_{13} \cap \ldots \cap H_{1 k}$ is a proper subset of the interior of the spherical polygon $H_{12} \cap$ $H_{13} \cap \ldots \cap H_{1, k-1}$ if and only if the bisector $B_{1 k}$ enters $H_{12} \cap H_{13} \cap \ldots \cap H_{1, k-1}$. In that case, the segment of $B_{1 k}$ that lies inside the larger polygon becomes part of the boundary of the smaller polygon.

\section{Regular polyhedra}

The regular polyhedra, which are the surfaces of the Platonic solids, have been studied since antiquity. Our interest here stems from the problem of spreading $m$ points on a sphere, uniformly in the sense that each point is equidistant from its nearest neighbors, that all such distances are equal, and that the points do not all lie in a plane. It is wellknown that there are exactly five values of $m$ for which this problem has a solution. If $m=4$, the points must be the vertices of a regular tetrahedron; for the other four cases, the points must be the vertices of an octahedron, cube, dodecahedron, and icosahedron, respectively. For each of these cases, we shall calculate the mean distance from a point on the sphere to the nearest vertex.

For any regular polyhedron $P$, let

$$
\begin{aligned}
m & =\text { number of vertices of } P ; \\
n & =\text { number of faces of } P ; \\
p & =\text { number of edges of the face polygons of } P ; \\
q & =\text { number of edges of } P \text { that meet at each vertex of } P ; \\
P^{\prime} & =\text { the polyhedron whose vertices are the centers of the faces of } P .
\end{aligned}
$$

Note that $P^{\prime}$ is a regular polyhedron, known as the dual of $P$. The dual of the tetrahedron is a tetrahedron; the cube and octahedron are a dual pair, as are the dodecahedron and icosahedron. Table 4 summarizes some of the main features of regular polyhedra.

Now moving from $P$ to its circumsphere, $\mathbb{S}$, let

$$
\begin{aligned}
O & =\text { the center of } \mathbb{S} ; \\
\mathcal{P} & =\text { projection of } P \text { from } O \text { onto } \mathbb{S} ; \\
\mathcal{P}^{\prime} & =\text { projection of } P^{\prime} \text { from } O \text { onto } \mathbb{S} .
\end{aligned}
$$

Let $A$ be a vertex of $\mathcal{P}$. The neighborhood $N(A)$, as defined in Section 7, has boundaries formed by the perpendicular bisectors (which are great circles of $\mathbb{S}$ ) of the $q$ edges of $\mathcal{P}$ 


\begin{tabular}{|l|r|r|r|r|l|}
\hline \multicolumn{1}{|c|}{$P$} & $m$ & $n$ & $p$ & $q$ & \multicolumn{1}{|c|}{$P^{\prime}$} \\
\hline tetrahedron & 4 & 4 & 3 & 3 & tetrahedron \\
\hline cube & 8 & 6 & 4 & 3 & octahedron \\
\hline octahedron & 6 & 8 & 3 & 4 & cube \\
\hline dodecahedron & 20 & 12 & 5 & 3 & icosahedron \\
\hline icosahedron & 12 & 20 & 3 & 5 & dodecahedron \\
\hline
\end{tabular}

Table 4 Regular polyhedra

that meet in $A$. Thus, $A$ is the center of a regular spherical $q$-gon - indeed, the interior of this $q$-gon is $N(A)$ - so that the mean distance from a point on $\mathbb{S}$ to the nearest vertex of $\mathcal{P}$ equals the mean distance from $A$ to $N(A)$, which equals the mean distance from $A$ to the interior of the right spherical triangle $A B C$ described in Section 6. As an example, when $\mathcal{P}$ is a cube, $N(A)$ is the interior of a spherical 3-gon, illustrated as $B D F$ in Fig. 2.

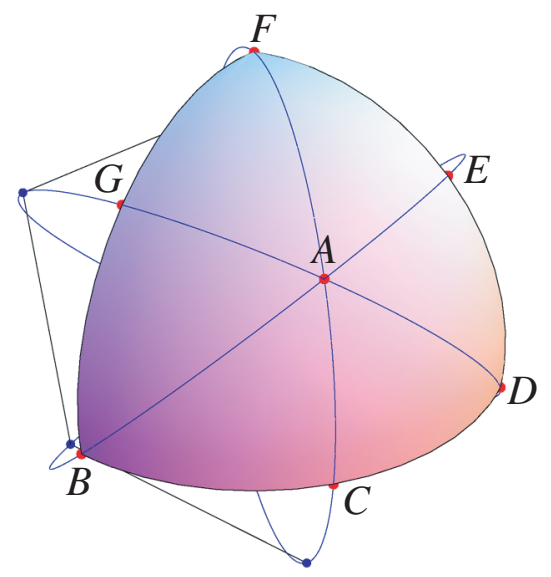

Fig. 2 The neighborhood of $A$ is the spherical triangle $B D F$

Recall from Section 6 that in the spherical right triangle $A B C$, we have $\measuredangle B A C=\pi / q$. This is the angle $\alpha$ in (5). We shall also need the angle $\beta=\measuredangle A B C$. Since $N(A)$ is one of the $n$ mutually congruent regular spherical polygons that comprise $\mathcal{P}^{\prime}$, the point $B$ is the vertex common to $p$ such polygons. Thus, the vertex angle at $B$ is $2 \pi / p$. Let $D$ and $F$ denote the vertices of $N(A)$ that are adjacent to $B$, so that $\measuredangle D B F=2 \pi / p$. The $\operatorname{arc} A B$ bisects angle $D B F$, so that $\beta=\pi / p$. For example, in Fig. $2, \measuredangle A B C=\pi / 4$.

The mean distance from $A$ to $N(A)$, given by (5) for a unit sphere, depends on the angle $\alpha$, sidelengths $b$ and $c$, and area $\mathcal{A}=\alpha+\beta-\pi / 2$. For the remainder of this section, we assume in each case that the regular polyhedron is prescribed in a unit sphere. Starting with $\alpha$ and $\beta$, the sidelengths are given (e.g., [4, p. 194]) by

$$
\cos a=\cos \alpha \csc \beta, \quad \cos b=\csc \alpha \cos \beta, \quad \cos c=\cot \alpha \cot \beta .
$$


We are now ready to apply (5) to each of the regular polyhedra.

Tetrahedron: $\alpha=\pi / 3, \beta=\pi / 3, \gamma=\pi / 2, \mathcal{A}=\pi / 6$. By (5), the mean distance from $A$ to $A B C$, and hence the mean distance from the unit sphere to the nearest vertex of the inscribed regular tetrahedron, is approximately 0.6907372805382.

Cube: $\alpha=\pi / 3, \beta=\pi / 4, \gamma=\pi / 2, \mathcal{A}=\pi / 12$. The mean distance from $A$ to $A B C$, and hence the mean distance from the unit sphere to the nearest vertex of the inscribed cube, is approximately 0.4907563907213 .

Octahedron: $\alpha=\pi / 4, \beta=\pi / 3, \gamma=\pi / 2, \mathcal{A}=\pi / 12$. The mean distance from $A$ to $A B C$, and hence the mean distance from the unit sphere to the nearest vertex of the inscribed regular octahedron is approximately 0.5667786396257.

Dodecahedron: $\alpha=\pi / 3, \beta=\pi / 5, \gamma=\pi / 2, \mathcal{A}=\pi / 30$. The mean distance from $A$ to $A B C$, and hence the mean distance from the unit sphere to the nearest vertex of the inscribed regular dodecahedron is approximately 0.3146937663893 .

Icosahedron: $\alpha=\pi / 5, \beta=\pi / 3, \gamma=\pi / 2, \mathcal{A}=\pi / 30$. The mean distance from $A$ to $A B C$, and hence the mean distance from the unit sphere to the nearest vertex of the inscribed regular icosahedron is approximately 0.38961615427.

To summarize, the five mean distances have been calculated from the angles $\alpha=\pi / p$ and $\beta=\pi / q$, where $p$ and $q$ are the number of edges of regular polygons associated with a polyhedron $P$, as described above. The pair $(q, p)$ is widely known as the Schläfli symbol of $P$, so that $(p, q)$ is the Schläfli symbol of the dual, $P^{\prime}$, of $P$.

A final comment is that the circumsphere of $P^{\prime}$ lies inside that of $P$, so that $\left(P^{\prime}\right)^{\prime} \neq P$, although $\left(P^{\prime}\right)^{\prime}$ is of course similar to $P$. On the other hand, $\left(\mathcal{P}^{\prime}\right)^{\prime}$ is not only similar to $\mathcal{P}$, but also $\left(\mathcal{P}^{\prime}\right)^{\prime}=\mathcal{P}$, so that we may certainly call $\mathcal{P}^{\prime}$ the dual of $\mathcal{P}$.

\section{Probabilistic interpretation}

The term "mean distance" can be interpreted as "expected distance" in the sense that this term is used in probability theory. This interpretation extends to a variety of considerations, but for present purposes, we assume that $\mathcal{R}$ is a cap or zone as in Section 3 . Let $P$ be a uniformly distributed random point [3] on $\mathcal{R}$. Let $N=(0,0,1)$, and let $X$ be the random variable given by

$$
X=\text { distance from } N \text { to } P \text {. }
$$

The expected value $E(X)$ is then the number $M$ in (4). The density function $f$ and distribution function $F$ are given by

$$
\begin{aligned}
& f(x)= \begin{cases}(\sin x) /(b-a) & \text { if } \arccos b \leq x \leq \arccos a \\
0 & \text { otherwise }\end{cases} \\
& F(x)= \begin{cases}0 & 0 \leq x<\arccos b \\
(b-\cos x) /(b-a) & \text { if } \arccos b \leq x \leq \arccos a \\
1 & \text { if } x>\arccos a\end{cases}
\end{aligned}
$$


Let $Y$ be the distance from the equator to $P$, so that $Y=\pi / 2-X$. From $F(x)$ we find the density function of $Y$ :

$$
g(y)= \begin{cases}(\cos y) /(b-a) & \text { if } \arcsin a \leq y \leq \arcsin b \\ 0 & \text { otherwise }\end{cases}
$$

The density functions $f$ and $g$ can be used to find the variances of $X$ and $Y$. For example, if $a=0$ and $b=1$ (so that $\mathcal{R}$ is the northern hemisphere), then $E(X)=1$ and $E(Y)=$ $\pi / 2-1$. The identity for variance, $\sigma_{X}^{2}=E\left(X^{2}\right)-[E(X)]^{2}$ yields $\sigma_{X}^{2}=\pi-3$, and similarly, $\sigma_{Y}^{2}=\pi-1$.

These findings may serve as a guide for probabilistic or statistical investigations involving computer samplings [3] of millions of points uniformly distributed on a sphere.

\section{Acknowledgement}

The author thanks the referee for pointing the way toward much simpler proofs. In the original manuscript, integrals were calculated as iterated integrals over regions in the $x y$-plane, and angles $\alpha$ and $\beta$ in Section 8 were laboriously found starting with explicit vertices, such as $( \pm 1, \pm 1, \pm 1)$ for the cube.

\section{References}

[1] "Spherical Triangle", http://www.walter-fendt.de/m14e/sphertriangle.htm

[2] Kells, L.M.: Plane and Spherical Trigonometry. 3rd edition, McGraw-Hill, New York 1951.

[3] MathWorld, "Sphere Point Picking", http://mathworld.wolfram.com/SpherePointPicking.html

[4] Palmer, C.I.; Leigh, C.W.; Kimball, S.H.: Plane and Spherical Trigonometry. 5th edition, McGraw-Hill, New York 1950

Clark Kimberling

University of Evansville

1800 Lincoln Avenue

Evansville, IN 47722, USA

e-mail: ck6@evansville.edu 